\title{
ASSISTÊNCIA DE ENFERMAGEM AO IDOSO: PROMOVENDO CUIDADO FRENTE AO RISCO DE QUEDAS
}

\author{
NURSING CARE FOR THE ELDERLY: PROMOTING CARE FACING THE \\ RISK OF FALLS
}

\author{
ATENCIÓN DE ENFERMERÍA PARA ANCIANOS: PROMOVER LA \\ ATENCIÓN ANTE EL RIESGO DE CAÍDAS
}

\author{
Bruno Henrique Souza Izidório ${ }^{1}$ \\ Ana Lessa Vilhena Emerick ${ }^{2}$ \\ Carolayne Moreira Brito ${ }^{3}$ \\ Gabriela Pereira Quintão ${ }^{4}$ \\ Larissa Silva Rodrigues ${ }^{5}$
}

RESUMO: Objetivo: Objetivamos investigar como e quais são as ações de enfermagem na assistência ao idoso sobre o risco de quedas. A ações executadas pela equipe de enfermagem auxiliam na prevenção, cuidado, recuperação e também facilita o reconhecimento dos grupos sujeitos a risco de quedas visando o que devemos implementar e quais intervenções adequadas devemos executar. Método: Realizamos uma análise baseada em uma pesquisa descritiva exploratória com base na pesquisa integrativa com abordagem qualitativa. Foram utilizados 12 artigos e compreendidos entre o período de 2008 a 2018. Os artigos e manuais foram selecionados através dos descritores nas bases de pesquisa Scielo e BVS. Resultados: A pesquisa demonstrou que é importante ter o conhecimento dos fatores de risco associados as quedas em idosos, para auxiliar na implantação de métodos e ações que aumentam a qualidade de vida e ajudam na prevenção de quedas em idosos. Conclusão: Devemos auxiliar, encaminhar e estarmos atentos aos riscos diários de cada paciente. É necessário um bom atendimento e acolhimento para que possamos atuar nessa prevenção e possibilitar uma qualidade de vida melhor para os idosos em relação aos riscos.

\footnotetext{
${ }^{I}$ Enfermeiro Mestrando pelo Centro Universitário Augusto Motta (UNISUAM), pós-graduação em Saúde da Família pela Faculdade Venda Nova do Imigrante (FAVENI), graduação em Enfermagem. Faculdade do Futuro (FAF), professor da Faculdade do Futuro (FAF), brunoizidorioo@outlook.com. ${ }^{2}$ Acadêmica do curso de graduação de Enfermagem da Faculdade do Futuro, analessaemerick@outlook.com.br

${ }^{3}$ Acadêmica do curso de graduação de Enfermagem da Faculdade do Futuro, carolaynemoreirabritoı6@gmail.com

${ }^{4}$ Acadêmica do curso de graduação de Enfermagem da Faculdade do Futuro, gabiquintaor9@gmail.com

${ }^{5}$ Acadêmica do curso de graduação de Enfermagem da Faculdade do Futuro, lariissa.rodriigues@hotmail.com
} 
Palavras-Chaves: Risco de quedas. Assistência de Enfermagem.

ABSTRACT: Objective: We aim to investigate how and what are the nursing actions in assisting the elderly about the risk of falls. The actions performed by the nursing team assist in prevention, care, recovery and also facilitate the recognition of groups subject to risk of falls, aiming at what we should implement and what appropriate interventions we should perform. Method: We performed an analysis based on an exploratory descriptive research based on the integrative research with a qualitative approach. Twelve articles were used and included between 2008 and 2018. The articles and manuals were selected through the descriptors in the Scielo and VHL research bases. Results: Research has shown that it is important to have knowledge of the risk factors associated with falls in the elderly, to assist in the implementation of methods and actions that increase the quality of life and help in the prevention of falls in the elderly.Conclusion: We must assist, refer and be aware of the daily risks of each patient. Good care and reception is necessary so that we can act in this prevention and enable a better quality of life for the elderly at risk.

Descriptors: Risk of falls Nursing Assistance.

RESUMEN: Objetivo: Nuestro objetivo es investigar cómo y cuáles son las acciones de enfermería para ayudar a los ancianos sobre el riesgo de caídas. Las acciones realizadas por el equipo de enfermería ayudan en la prevención, atención, recuperación y también facilitan el reconocimiento de grupos sujetos a riesgo de caídas, con el objetivo de lo que debemos implementar y qué intervenciones apropiadas debemos realizar. Método: Realizamos un análisis basado en una investigación exploratoria descriptiva basada en la investigación integradora con un enfoque cualitativo. Se utilizaron doce artículos que se incluyeron entre 2008 y 2018. Los artículos y manuales se seleccionaron a través de los descriptores en las bases de investigación de Scielo y BVS. Resultados: La investigación ha demostrado que es importante tener conocimiento de los factores de riesgo asociados con las caídas en los ancianos, para ayudar en la implementación de métodos y acciones que aumenten la calidad de vida y ayuden a prevenir las caídas en los ancianos. Conclusión: debemos ayudar, derivar y estar conscientes de los riesgos diarios de cada paciente. Es necesario un buen cuidado y recepción para que podamos actuar en esta prevención y permitir una mejor calidad de vida para las personas mayores en riesgo.

Descriptores: Riesgo de caídas; Asistencia de enfermería.

\section{INTRODUÇÃO}

No Brasil, como em outros países em desenvolvimento, vem tendo um crescimento importante no aumento populacional de pessoas com 60 anos ou mais, 
isso faz com que tenha uma grande demanda nos serviços de saúde, afetando deste modo, a assistência prestada (FREITAS et al., 20II).

A população está envelhecendo, o Brasil em 2025, será o sexto país com maior número de idosos, com um aumento de 16 vezes mais dessa população (MIRANDA, ATHAYDE e BARBOSA, 2018). Para Pinho et al., (2012), o envelhecimento relacionado a queda é uma grande preocupação coletiva, gerando um grande problema para saúde pública. Outra grande dificuldade na atenção aos idosos é conseguir oferecer uma boa qualidade de vida e independência.

As situações comuns no processo de envelhecimento têm sido ocasionadas por alterações na diminuição da força muscular, amplitude dos movimentos, dificuldade de locomoção, sensibilidade auditiva e visual (PINHO et al., 20I2). Dentre as dificuldades desses problemas podemos ressaltar as quedas, que ocorrem em sua própria casa durante atividades do dia a dia como subir e descer escadas, ir ao banheiro ou trabalhar na cozinha. É uma ocorrência comum e muito temível pela maioria dos idosos, essas ocorrências representam a primeira causa de acidentes pela população idosa (PINHO et al., 2012).

Para Beck et al., (20II), "Queda é definida como um evento não intencional que tem como resultado a mudança de posição do indivíduo para um nível mais baixo em relação à sua postura inicial". A ocorrência de quedas vem se tornando um dos principais problemas de saúde pública e que contribuem para inaptidão dos idosos (BECK et al., 20II).

De acordo com a Organização Mundial da Saúde a queda é uma das complicações mais significativas e comuns entre os idosos e aumenta cada vez mais com o avanço da idade tanto no sexo feminino quanto no sexo masculino (BECK et al., 2011).

Os fatores de risco podem ser separados em dois grupos: intrínsecos e extrínsecos. Os fatores intrínsecos estão relacionados aos comportamentos físicos e mentais que acarretam grandes mudanças nas situações de envelhecimento, como a diminuição da capacidade funcional, perda da força muscular, aparecimento de doenças crônicas, doenças osteoarticulares, Doença de Parkison, alterações do 
equilíbrio, distúrbios de percepção ambiental, inatividade, alterações na visão e audição ou até mesmo pelo uso de medicamentos (PINHO et al., 2012).

Para Freitas et al., (2011), os fatores extrínsecos são ocasionados por fatores ambientais, evidenciados pelas quedas e frequentemente, envolvem situações do dia a dia. Podemos citar dentre os fatores ambientais a iluminação inadequada, presença de obstáculos, ausência de corrimãos e adaptações em escadas e banheiros, pisos escorregadios, calçados inadequados, superfícies irregulares, presença de tapetes e degraus pela casa, entre outros (PINHO et al., 2012). As quedas geram complicações para a saúde, como o aumento do tempo da internação e custo com tratamento. (PINHO et al., 2012).

A maior parte das ações executadas são pela equipe de enfermagem que tem um papel muito importante no cuidado direto com paciente, facilitando o reconhecimento dos grupos sujeitos a risco de quedas, o Enfermeiro deve implementar ações e intervenções adequadas como orientar aos familiares sobre a importância de oferecer um ambiente seguro para proporcionar uma boa qualidade de vida, visando à prevenção, ou mesmo a diminuição ou o fim dessas ocorrências (LUZIA, ALMEIDA e LUCENA, 2014).

O objetivo deste estudo foi avaliar o risco de quedas em idosos, identificar suas causas, ações e o reconhecimento dos grupos sujeitos a esses riscos, visando o que devemos implementar e quais Intervenções de Enfermagem adequadas devemos executar para a prevenção e a redução dos riscos de quedas em idosos.

Como a Assistência de Enfermagem e os cuidados podem auxiliar na prevenção e diminuição dos riscos de quedas em idosos?

\section{MÉTODO}

O estudo foi baseado em uma pesquisa descritiva exploratória com base na pesquisa integrativa com abordagem qualitativa, e que inclui interpretação dos dados contidos em cada texto dos artigos, correspondendo a uma leitura direcionada e organizada pelos fichamentos dos textos. 
Para o desenvolvimento desse estudo foram utilizados 12 artigos e compreendidos entre o período de 2008 a 2018. Os artigos e manuais foram selecionados através dos descritores (DESC) nas bases de pesquisa Scielo (Scientific Eletronic Library Online) e BVS (Biblioteca Virtual em Saúde).

Esta pesquisa dividiu-se em várias etapas. Inicialmente procedeu-se com a leitura dos artigos, depois os artigos foram separados em eixos para o levantamento de dados bibliográficos sobre o tema da pesquisa, abrangendo a escolha do tema, a formulação do problema, a leitura e fichamento dos textos, a justificativa e a relevância da pesquisa, para que pudesse ser realizada a elaboração dos resultados e discussões.

\section{RESULTADOS}

Tabela I - Artigos levantados nas bases de dados BVS e Scielo sobre revisão integrativa.

\begin{tabular}{|c|c|c|c|c|}
\hline $\begin{array}{l}\text { Procedênci } \\
\text { a }\end{array}$ & Título do artigo & Autores & $\begin{array}{l}\text { Periódico (vol, no, pág, } \\
\text { ano) }\end{array}$ & $\begin{array}{l}\text { Considerações / } \\
\text { Temática }\end{array}$ \\
\hline $\begin{array}{l}\text { BVS } \\
\text { (Biblioteca } \\
\text { Virtual em } \\
\text { Saúde) }\end{array}$ & $\begin{array}{l}\text { Cair faz parte da } \\
\text { vida: Fatores de risco } \\
\text { para quedas em } \\
\text { idosos. }\end{array}$ & $\begin{array}{l}\text { Stamm B, Leite MT, } \\
\text { Hildebrandt LM, } \\
\text { Kirchner RM, } \\
\text { Menezes LP. }\end{array}$ & $\begin{array}{l}\text { J. res.: fundam. care. } \\
\text { online. } 8(4), \text { p. 508o-5086, } \\
\text { out./dez } 2016 .\end{array}$ & $\begin{array}{l}\text { Determina a } \\
\text { identificação } \\
\text { da prevalência } \\
\text { de quedas em } \\
\text { idosos, } \\
\text { principalment } \\
\text { e residentes } \\
\text { em áreas } \\
\text { urbanas, onde } \\
\text { buscam } \\
\text { analisar os } \\
\text { fatores de risco } \\
\text { associados a } \\
\text { queda. }\end{array}$ \\
\hline $\begin{array}{l}\text { Scielo } \\
\text { (Scientific } \\
\text { Eletronic } \\
\text { Library } \\
\text { Online) }\end{array}$ & $\begin{array}{l}\text { Avaliação do risco de } \\
\text { quedas e fatores } \\
\text { associados em idosos. }\end{array}$ & Reis LA, Flôres CMR. & $\begin{array}{l}\text { Revista Baiana de } \\
\text { Enfermagem, v. } 28 \text {, n. I, p. } \\
42-49 \text {, jan./abr 2014. }\end{array}$ & $\begin{array}{l}\text { Analisa o risco } \\
\text { de queda e os } \\
\text { fatores } \\
\text { associados a } \\
\text { idoso em } \\
\text { frequentadores } \\
\text { de grupos de } \\
\text { convivência. }\end{array}$ \\
\hline $\begin{array}{l}\text { BVS } \\
\text { (Biblioteca } \\
\text { Virtual em } \\
\text { Saúde) }\end{array}$ & $\begin{array}{l}\text { Quedas em idosos: } \\
\text { Avaliação dos fatores } \\
\text { de risco. }\end{array}$ & $\begin{array}{l}\text { Sardinha AHL, } \\
\text { Cantanhêde NLC. }\end{array}$ & $\begin{array}{l}\text { Revista Nursing. v. 21, } \\
\text { n. 240, p. } 2160-2163, \\
\text { jun./jan 2018. }\end{array}$ & $\begin{array}{l}\text { Avalia os } \\
\text { fatores de } \\
\text { quedas em } \\
\text { idosos, } \\
\text { desenvolvido } \\
\text { no Centro de } \\
\text { Saúde da } \\
\text { Liberdade. } \\
\text { Busca ilustrar } \\
\text { a realidade dos } \\
\text { idosos sobra os } \\
\text { fatores de risco } \\
\text { de queda. }\end{array}$ \\
\hline
\end{tabular}




\begin{tabular}{|c|c|c|c|c|}
\hline $\begin{array}{l}\text { Scielo } \\
\text { (Scientific } \\
\text { Eletronic } \\
\text { Library } \\
\text { Online) }\end{array}$ & $\begin{array}{l}\text { Cuidado de } \\
\text { Enfermagem para } \\
\text { prevenção de quedas } \\
\text { em idosos: Proposta } \\
\text { para ação. }\end{array}$ & $\begin{array}{l}\text { Freitas R, Santos SSC, } \\
\text { Hammerschmidt KSA, } \\
\text { Silva ME, Pelzer MT. }\end{array}$ & $\begin{array}{l}\text { Rev. Bras. Enferm. v. } 64 \text {, } \\
\text { n. } 3 \text {, p. } 478-485 \text {, mai./jun } \\
\text { 20II. }\end{array}$ & $\begin{array}{l}\text { Trata de uma } \\
\text { construção de } \\
\text { propostas de } \\
\text { ação de } \\
\text { enfermagem } \\
\text { para prevenção } \\
\text { de quedas em } \\
\text { idosos. }\end{array}$ \\
\hline $\begin{array}{l}\text { BVS } \\
\text { (Biblioteca } \\
\text { Virtual em } \\
\text { Saúde) }\end{array}$ & $\begin{array}{l}\text { Queda de Idosos: } \\
\text { Desvelando situações } \\
\text { de vulnerabilidade. }\end{array}$ & $\begin{array}{l}\text { Luzardo AR, Júnior } \\
\text { NFP, Medeiros M, } \\
\text { Lima LSB, Wolkers } \\
\text { PCB, Santos SMA. }\end{array}$ & $\begin{array}{l}\text { Rev. Min. Enferm. v. 2I, } \\
\text { p. I025-1032, fev./jul 2017. }\end{array}$ & $\begin{array}{l}\text { Destaca como } \\
\text { o } \\
\text { envelheciment } \\
\text { o populacional } \\
\text { impacta } \\
\text { situações de } \\
\text { vulnerabilidad } \\
\text { e a que idosos } \\
\text { estão expostos. }\end{array}$ \\
\hline $\begin{array}{l}\text { Scielo } \\
\text { (Scientific } \\
\text { Eletronic } \\
\text { Library } \\
\text { Online) }\end{array}$ & $\begin{array}{l}\text { Fatores de Risco que } \\
\text { contribuem para } \\
\text { queda em idosos. }\end{array}$ & $\begin{array}{l}\text { Miranda AP, Athayde } \\
\text { IF, Barbosa MEI. }\end{array}$ & $\begin{array}{l}\text { Revista Nursing, v. 21, } \\
\text { n. } 238 \text {, p. 2063-2067, } \\
\text { out./jan 2018. }\end{array}$ & $\begin{array}{l}\text { Identifica os } \\
\text { fatores que } \\
\text { ocasionam a } \\
\text { queda nos } \\
\text { idosos, } \\
\text { considerando } \\
\text { as } \\
\text { consequências } \\
\text { e descrevendo } \\
\text { as mudanças } \\
\text { ocorridas na } \\
\text { vida diária dos } \\
\text { idosos. }\end{array}$ \\
\hline $\begin{array}{l}\text { Scielo } \\
\text { (Scientific } \\
\text { Eletronic } \\
\text { Library } \\
\text { Online) }\end{array}$ & $\begin{array}{l}\text { Mapeamento de } \\
\text { cuidados de } \\
\text { enfermagem para } \\
\text { pacientes com risco } \\
\text { de quedas na Nursing } \\
\text { Interventions } \\
\text { Classification. }\end{array}$ & $\begin{array}{l}\text { Luzia MF, Almeida } \\
\text { MA, Lucena AF. }\end{array}$ & $\begin{array}{l}\text { Rev. Esc. Enferm. USP, v. } \\
48, \text { n. } 4 \text {, p. } 632-639 \\
\text { nov./jun } 2014 .\end{array}$ & $\begin{array}{l}\text { Destaca a } \\
\text { identificação } \\
\text { dos cuidados } \\
\text { de } \\
\text { enfermagem } \\
\text { prescritos para } \\
\text { pacientes } \\
\text { hospitalizados } \\
\text { com risco de } \\
\text { quedas para } \\
\text { comparação } \\
\text { com as } \\
\text { intervenções } \\
\text { da Nursing } \\
\text { Interventions } \\
\text { Classification. }\end{array}$ \\
\hline $\begin{array}{l}\text { Scielo } \\
\text { (Scientific } \\
\text { Eletronic } \\
\text { Library } \\
\text { Online) }\end{array}$ & $\begin{array}{l}\text { Qualidade de vida em } \\
\text { Idosos que sofreram } \\
\text { quedas: Revisão } \\
\text { Integrativa da } \\
\text { Literatura. }\end{array}$ & $\begin{array}{l}\text { Nicolussi AC, Fhon } \\
\text { JRS, Santos CAV, } \\
\text { Kusomota L, Marques } \\
\text { S, Rodrigues RAP. }\end{array}$ & $\begin{array}{l}\text { Ciência e Saúde Coletiva, } \\
\text { v. 17, n. 3, p. 723-730, } \\
\text { jun./set 2oro. }\end{array}$ & $\begin{array}{l}\text { Buscou avaliar } \\
\text { o } \\
\text { conhecimento } \\
\text { cientifico } \\
\text { produzido } \\
\text { relacionado a } \\
\text { qualidade de } \\
\text { vida do idoso } \\
\text { que sofreu } \\
\text { queda. }\end{array}$ \\
\hline $\begin{array}{l}\text { Scielo } \\
\text { (Scientific } \\
\text { Eletronic } \\
\text { Library } \\
\text { Online) }\end{array}$ & $\begin{array}{l}\text { Risco de quedas em } \\
\text { idosos: Revisão } \\
\text { integrativa pelo } \\
\text { diagnóstico da North } \\
\text { American Nursing } \\
\text { Diagnosis Association. }\end{array}$ & $\begin{array}{l}\text { Santos SSC, Silva ME, } \\
\text { Pinho LB, Gautério } \\
\text { DP, Pelzer MT, } \\
\text { Silveira RS. }\end{array}$ & $\begin{array}{l}\text {, Rev. Esc. Enferm. USP, } \\
\text { v. 46, n. 5, p. 1227-1236, } \\
\text { jul./fev } 2012 .\end{array}$ & $\begin{array}{l}\text { Analisa uma } \\
\text { produção } \\
\text { cientifica de } \\
\text { fatores de risco } \\
\text { para quedas, a } \\
\text { partir do } \\
\text { diagnótico da } \\
\text { North } \\
\text { American } \\
\text { Nursing } \\
\text { Diagnosis }\end{array}$ \\
\hline
\end{tabular}




\begin{tabular}{|c|c|c|c|c|}
\hline & & & & $\begin{array}{l}\text { Association, na } \\
\text { literatura } \\
\text { cientifica } \\
\text { brasileira e } \\
\text { estrangeira, de } \\
2005 \text { a 2010. }\end{array}$ \\
\hline $\begin{array}{l}\text { Scielo } \\
\text { (Scientific } \\
\text { Eletronic } \\
\text { Library } \\
\text { Online) }\end{array}$ & $\begin{array}{l}\text { Estudo da presença } \\
\text { de fatores de riscos } \\
\text { intrínsecos para } \\
\text { quedas, em idosos } \\
\text { institucionalizados. }\end{array}$ & $\begin{array}{l}\text { Menezes RL, Bachion } \\
\text { MM. }\end{array}$ & $\begin{array}{l}\text { Ciência e Saúde Coletiva, } \\
\text { v. I3, n. 4, p. I209-1218, } \\
\text { dez./jul 2008. }\end{array}$ & $\begin{array}{l}\text { Objetivou a } \\
\text { identificar a } \\
\text { presença de } \\
\text { fatores } \\
\text { intrínsecos que } \\
\text { predipõem a } \\
\text { quedas em } \\
\text { idosos } \\
\text { moradores em } \\
\text { instituições de } \\
\text { longa } \\
\text { permanência. }\end{array}$ \\
\hline $\begin{array}{l}\text { Scielo } \\
\text { (Scientific } \\
\text { Eletronic } \\
\text { Library } \\
\text { Online) }\end{array}$ & $\begin{array}{l}\text { Fatores associados às } \\
\text { quedas entre idosos } \\
\text { praticantes de } \\
\text { atividades físicas. }\end{array}$ & $\begin{array}{l}\text { Beck AP, Antes DL, } \\
\text { Meurer ST, Benedetti } \\
\text { TR, Lopes MA. }\end{array}$ & $\begin{array}{l}\text { Texto \& Contexto } \\
\text { Enferm. } \\
\text { v. 2o, n. 2, p. 28o-286, } \\
\text { abr./jun 2olr. }\end{array}$ & $\begin{array}{l}\text { Analisa a } \\
\text { incidência e os } \\
\text { fatores } \\
\text { associados às } \\
\text { quedas em } \\
\text { idosos } \\
\text { praticantes de } \\
\text { atividades } \\
\text { físicas. }\end{array}$ \\
\hline $\begin{array}{l}\text { Scielo } \\
\text { (Scientific } \\
\text { Eletronic } \\
\text { Library } \\
\text { Online) }\end{array}$ & $\begin{array}{l}\text { Avaliação do risco de } \\
\text { quedas em idosos } \\
\text { atendidos em } \\
\text { Unidade Básica de } \\
\text { Saúde. }\end{array}$ & $\begin{array}{l}\text { Pinho TAM, Silva } \\
\text { AO, Tura LFR, } \\
\text { Moreira MASP, } \\
\text { Gurgel SN, Smith } \\
\text { AAF, Bezerra VP. }\end{array}$ & $\begin{array}{l}\text { Rev. Esc. Enferm. USP, } \\
\text { v. 46, n. 2, p. } 320-327 \\
\text { mai./jul 2012. }\end{array}$ & $\begin{array}{l}\text { Avalia o } \\
\text { envelheciment } \\
\text { o de forma } \\
\text { abrupta } \\
\text { representando } \\
\text { um grande } \\
\text { desafio para os } \\
\text { orgãos } \\
\text { competentes, } \\
\text { que por sua } \\
\text { vez, } \\
\text { necessitam de } \\
\text { novas políticas } \\
\text { públicas de } \\
\text { saúde, } \\
\text { inclusive na } \\
\text { preveção de } \\
\text { quedas. }\end{array}$ \\
\hline
\end{tabular}

O artigo "Cair faz parte da vida: Fatores de risco para quedas em idosos", do ano de 2016, foi publicado do Journal of Research: Fundamental Care Online da base de pesquisa BVS que determina a identificação da prevalência de quedas em idosos, principalmente residentes em áreas urbanas, onde buscam analisar os fatores de risco associados a queda. Os artigos "Avaliação do risco de quedas e fatores associados em idosos" e "Mapeamento de cuidados de enfermagem para pacientes com risco de quedas na Nursing Interventions Classification”, são do ano de 2014 e ambos são da base de pesquisa Scielo e foram publicados na Revista Baiana de Enfermagem e Revista da 
Escola de Enfermagem da USP respectivamente, o primeiro analisa o risco de queda e os fatores associados a idoso em frequentadores de grupos de convivência, já o segundo destaca a identificação dos cuidados de enfermagem prescritos para pacientes hospitalizados com risco de quedas para comparação com as intervenções da Nursing Interventions Classification.

Os artigos "Quedas em idosos: Avaliação dos fatores de risco" e "Fatores de Risco que contribuem para queda em idosos”, são de 2018 e ambos foram publicados na Revista Nursing, o primeiro é da base de pesquisa BVS que avalia os fatores de quedas em idosos, desenvolvido no Centro de Saúde da Liberdade e busca ilustrar a realidade dos idosos sobra os fatores de risco de queda, o segundo é da base de pesquisa Scielo que identifica os fatores que ocasionam a queda nos idosos, considerando as consequências e descrevendo as mudanças ocorridas na vida diária dos idosos. Os artigos "Cuidado de Enfermagem para prevenção de quedas em idosos: Proposta para ação" e "Fatores associados às quedas entre idosos praticantes de atividades físicas”, ambos são de 201 e da base de pesquisa Scielo, o primeiro foi publicado na Revista Brasileira de Enfermagem e que trata de uma construção de propostas de ação de enfermagem para prevenção de quedas em idosos, o segundo foi publicado na Revista Texto \& Contexto de Enfermagem que analisa a incidência e os fatores associados às quedas em idosos praticantes de atividades físicas.

O artigo "Queda de Idosos: Desvelando situações de vulnerabilidade", do ano de 2017 e foi publicado na Revista Mineira de Enfermagem e encontrado na base de pesquisa BVS que destaca como o envelhecimento populacional impacta situações de vulnerabilidade a que idosos estão expostos. "Qualidade de vida em Idosos que sofreram quedas: Revisão Integrativa da Literatura" do ano de 20ı, foi publicado na Revista Ciência e Saúde coletiva da base de pesquisa Scielo que buscou avaliar o conhecimento cientifico produzido relacionado a qualidade de vida do idoso que sofreu queda. Os artigos "Risco de quedas em idosos: Revisão integrativa pelo diagnóstico da North American Nursing Diagnosis Association" e "Avaliação do risco de quedas em idosos atendidos em Unidade Básica de Saúde” ambos foram publicados em 2012 pela Revista da Escola de Enfermagem da USP da base de pesquisa Scielo, o primeiro analisa uma produção cientifica de fatores de risco para quedas, a partir do 
diagnótico da North American Nursing Diagnosis Association, na literatura cientifica brasileira e estrangeira, de 2005 a 20I0, o segundo avalia o envelhecimento de forma abrupta representando um grande desafio para os orgãos competentes, que por sua vez, necessitam de novas políticas públicas de saúde, inclusive na prevenção de quedas. "Estudo da presença de fatores de riscos intrínsecos para quedas, em idosos institucionalizados", foi publicado no ano de 2008 pela Revista Ciência e Saúde coletiva da base de pesquisa Scielo e objetivou a identificar a presença de fatores intrínsecos que predispõem a quedas em idosos moradores em instituições de longa permanência.

\section{DISCUSSÃO}

\section{Assistência de Enfermagem voltada ao cuidado}

Segundo Freitas et al., (2011), a proposta para o cuidado de enfermagem busca intervir na prevenção de quedas, realizando a promoção de saúde dos idosos através de trabalhos preventivos adotando medidas e cuidados para diminuição dos fatores de risco associados. O autor ainda destaca a importância dos cuidados de Enfermagem que auxiliam na promoção das habilidades funcionais motoras e cognitivas do idoso.

Para Luzia, Almeida e Lucena (2014), destaca alguns cuidados de enfermagem importantes para a prevenção de risco de quedas principalmente em manter as grades no leito, orientar paciente e família quanto aos riscos e prevenções de quedas, manter a campainha ao alcance do paciente e manter pertences próximos ao paciente.

De certo modo, é relevante ressaltar a necessidade de um cuidado humanizado voltado ao ideal do paciente, prestando assistência, em decorrência da importância cabível referente a questões relacionadas ao risco de quedas, de modo que, o enfermeiro está capacitado a reverter por meio de intervenções básicas, situações que envolvem o risco que pode levar a queda e maiores consequências para o paciente.

Outros principais cuidados que podemos citar são sobre as orientações ao paciente e a família, quanto sua mobilização e deambulação, tanto como segurança do ambiente e seu monitoramento de alterações neurológicas. É importante ressaltar que 
um diálogo entre enfermeiros e idosos tem sido uma experiência intersubjetiva e devem ancorados nas necessidades do idoso (FREITAS et al., 2010).

\section{Risco de Quedas: Avaliação preventiva e qualitativa}

Para Stamm et al., (2016), é importante ter o conhecimento dos fatores de risco associados as quedas em idosos, para auxiliar na implantação de métodos e ações que aumentam a qualidade de vida e ajudam na prevenção de quedas em idosos.

O risco de quedas entre os idosos avaliados era elevado, contendo um risco maior associado a idosos do sexo feminino que geralmente são viúvas ou contém algum problema de saúde (REIS e FLÔRES, 2014).

Para Sardinha e Cantanhêde (2018) é importante realizar medidas preventivas para identificar ou avaliar os riscos de quedas em idosos, para que sejam implantados protocolos específicos de fatores de risco de quedas, como por exemplo a "Escala de risco de queda de Downton" que facilmente pode ser aplicado em idosos. Um dos fatores de grande importância para a saúde pública é a queda em idosos que gera várias consequências para pessoas deste grupo, sendo de causa multifatorial, estabelecendo fatores extrínsecos e intrínsecos evidenciado por uma alta de complexidade terapêutica de complicada prevenção, é preciso uma abordagem multidisciplinar e uma assistência regular a saúde do idoso voltadas a estratégia de saúde da família (MIRANDA, ATHAYDE e BARBOSA, 2018).

Segundo Santos et al., (2012), é fundamental que se tenha um conhecimento aos fatores de risco ambientais, ressaltando obstáculos e condições ambientais que causam acidentes por quedas como recinto com móveis, objetos ou tapetes espalhados pelo chão, pouca iluminação, condições climáticas e pisos escorregadios. Ainda para o autor os riscos cognitivos relacionados ao estado mental rebaixado, caracterizada pelas demências recorrentes que envolve mudanças na cognição, físicas e comportamentais.

Os riscos fisiológicos são compostos por equilíbrio prejudicado, dificuldades visuais, dificuldades na marcha, incontinência e neoplasia (SANTOS et al., 2012). 
Para Menezes e Bachion (2008), as necessidades de avaliação pelos profissionais geriátricos podem promover a saúde do idoso, prevenindo agravos e complicações clínicas. Informações obtidas podem ser essenciais para o estabelecimento de marcadores que maximizam possibilidades dos eventos de quedas em idosos acontecerem.

Para uma avaliação dos riscos de quedas em idosos é importante uma realização de medidas preventivas promovendo uma conscientização da população, proporcionando uma qualidade de vida aos idosos reduzindo assim gastos com tratamentos ocasionados por quedas (PINHO et al., 2012).

\section{Quedas em Idosos e a tendência a maiores consequências}

Segundo Beck et al., (2011), é importante que seja estimulada a prática de atividades físicas, promovendo melhorias das capacidades funcionais dos idosos, estabelecendo exercícios que simulem as atividades diárias desses idosos além dos exercícios físicos normais, ocasionando uma diminuição dos riscos de quedas.

A partir de situações visíveis a curto prazo, o enfermeiro pode e se torna capacitado a promover cuidados que facilitem o bem-estar geral do paciente, de modo que, o mesmo consiga desenvolver sua saúde de forma única e plausível, e que situações de risco não interfiram nesse processo.

Cabe aos profissionais de saúde, principalmente aos enfermeiros de maior atuação, promover intervenções para a prevenção de quedas, podendo ser colocados em prática possibilitando uma qualidade de vida aos idosos (NICOLUSSI et al., 2010). Intervenções essas, objetivas, tal como uma simples retirada de mobílias que interfiram na movimentação, porém, algumas mobílias, podem auxiliar na locomoção, assim com a experiencia, formação e conhecimento, o enfermeiro consegue destacar tais prós e contras e desenvolver um cuidado específico voltado a qualidade de vida do paciente.

Para Luzardo et al., (2017), os serviços de saúde devem estar de prontidão para atuarem em casos de quedas, por meio de recursos físicos e humanos.

A capacitação dos profissionais é necessária para um bom atendimento de pessoas idosas vulneráveis a queda, auxiliando na prevenção do mesmo. 


\section{CONCLUSÃO}

A prevenção de quedas é tarefa difícil devido à fatores que as predispõe. Os fatores podem estar relacionados ao próprio envelhecimento com a diminuição dos reflexos e, também, relacionado a diversas doenças como a osteoporose, a Doença de Parkinson, o Alzheimer, e até mesmo pelo uso de medicamentos.

Essas causas precisam de cuidado integral. Através do profissional enfermeiro, consegue-se avaliar situações de risco, levantando problemas singulares, criando intervenções passíveis de conclusão, e, por fim, observar o resultado que se espera alcançar. Isso simplesmente me determina uma SAE (Sistematização da Assistência da Enfermagem), que, quando executada e promovida com excelência, consegue dispor de certezas em qualidade de promoção a saúde.

Concluímos que a equipe de enfermagem tem esse papel do cuidado, bem voltada a um atendimento capacitativo, cujo ato se torna importante nessa prevenção. Devemos auxiliar, encaminhar e estarmos atentos aos riscos diários de cada paciente. É necessário um bom atendimento e acolhimento para que possamos atuar nessa prevenção e possibilitar uma qualidade de vida melhor para os idosos em risco.

\section{REFERÊNCIAS}

I. STAMM B.; LEITE M. T.; HILDEBRANDT L. M.; KIRCHNER R. M.; MENEZES L. P. Cair faz parte da vida: Fatores de risco para quedas em idosos. J. Res. Fundam. Care. Online. v. 8, n. 4, p. 5080-5086, out./dez 2016.

2. REIS L. A.; FLÔRES C. M. R. Avaliação do risco de quedas e fatores associados em idosos. Revista Baiana de Enfermagem. v. 28, n. I, p. 42-49, jan./abr 2014.

3. SARDINHA A. H. L.; CANTANHÊDE N. L. C. Quedas em idosos: Avaliação dos fatores de risco. Revista Nursing. v. 2I, n. 240, p. 2160-2163, jun./jan 2018.

4. FREITAS R.; SANTOS S. S. C.; HAMMERSCHMIDT K. S. A.; SILVA M. E.; PELZER M. T. Cuidado de Enfermagem para prevenção de quedas em idosos: Proposta para ação. Rev. Bras. Enferm. v. 64, n. 3, p. 478-485, mai./jun 2011. 
5. LUZARDO A. R.; JÚNIOR N. F. P.; MEDEIROS M.; LIMA L. S. B.; WOLKERS P. C. B.; SANTOS S. M. A. Queda de Idosos: Desvelando situações de vulnerabilidade. Rev. Min. Enferm. v. 2I, p. 1025-1032, fev./jul 2017.

6. MIRANDA A. P.; ATHAIDE I. F.; BARBOSA M. E. I. Fatores de Risco que contribuem para queda em idosos. Revista Nursing, v. 21, n. 238, p. 2063-2067, out./jan 2018.

7. LUZIA M. F.; ALMEIDA M. A.; LUCENA A. F. Mapeamento de cuidados de enfermagem para pacientes com risco de quedas na Nursing Interventions Classification. Rev. Esc. Enferm. USP, v. 48, n. 4, p. 632-639, nov./jun 2014.

8. NICOLUSSI A. C.; FHON J. R. S.; SANTOS C. A. V.; KUSUMOTA L.; MARQUES S.; RODRIGUES R. A. P. Qualidade de vida em Idosos que sofreram quedas: Revisão Integrativa da Literatura. Ciência e Saúde Coletiva, v. 17, n. 3, p. 723730, jun./set 2010.

9. SANTOS S. S. C.; SILVA M. E.; PINHO L. B.; GAUTÉRIO D. P.; PELZER M. T.; SILVEIRA R. S. Risco de quedas em idosos: Revisão integrativa pelo diagnóstico da North American Nursing Diagnosis Association. Rev. Esc. Enferm. USP, v. 46, n. 5, p. 1227-1236, jul./fev 2012.

ro. MENEZES R. L.; BACHION M. M. Estudo da presença de fatores de riscos intrínsecos para quedas, em idosos institucionalizados. Ciência e Saúde Coletiva, v. 13, n. 4, p. 1209-1218, dez./jul 2008.

Ir. BECK A. P.; ANTES D. L.; MEURER S. T.; BENEDETTI T. R.; LOPES M. A. Fatores associados às quedas entre idosos praticantes de atividades físicas. Texto \& Contexto Enferm. v. 20, n. 2, p. 280-286, abr./jun 2011.

12. PINHO T. A. M.; SILVA A. O.; TURA L. F. R.; MOREIRA M. A. S. P.; GURGEL S. N.; SMITH A. A. F.; BEZERRA V. P. Avaliação do risco de quedas em idosos atendidos em Unidade Básica de Saúde. Rev. Esc. Enferm. USP, v. 46, n. 2, p. 320-327, mai./jul 2012. 